EPiC Series in Computing
Volume 56, 2018, Pages 23-34
$\begin{gathered}\text { Proceedings of the 5th International } \\ \text { OMNeT++ Community Summit }\end{gathered}$

\title{
Community-based Mobility Model and Probabilistic ORBIT Mobility Model Implementations in OMNeT++
}

\author{
Vishnupriya Kuppusamy, Leonardo Sarmiento, Asanga Udugama, and \\ Anna Förster \\ Sustainable Communication Networks, University of Bremen, Bremen, Germany \\ \{vp, adu, annafoerster\}@comnets. uni-bremen.de, sarmileo@uni-bremen.de
}

\begin{abstract}
Simulations of Opportunistic Networking (OppNet) protocols require the use of suitable synthetic mobility models or real world traces. Many synthetic mobility models have been proposed based on the study of human mobility individually and in groups. Opportunistic Protocol Simulator (OPS) is a budding simulator which is based on OMNeT++ to simulate OppNets. However, compared to other OppNet simulators in the literature, only very few synthetic mobility models exist in OMNeT ++ currently, restricting the simulation of OppNets to using the existing mobility models or traces. In this paper, we develop two more synthetic mobility models in $\mathrm{OMNeT}++$ namely community-based mobility model and probabilistic ORBIT based mobility, which can enhance the simulating environment available for OppNets in OMNeT++.
\end{abstract}

\section{Introduction}

Opportunistic Networks (OppNets) enable the nodes to communicate without the need for an infrastructure [1]. The nodes communicate directly with each other whenever they are in the connection range of each other. These networks were initially proposed to be used only in challenging environments like disaster scenarios and areas with lack of infrastructure development. However, they have the potential to cater to a variety of highly scalable wireless networks such as Internet of Things (IoT).

Performance evaluations of OppNets consist of two main components; the working of forwarding protocols themselves and the evaluation of these protocols for a highly scalable and dynamic network like OppNets. The dynamic nature of OppNets is highly influenced by the mobility of the nodes. Owing to the scalability and mobility of OppNets, evaluating their performance analysis in a large-scale real-world environment is still a challenge. Real testbeds often mirror the real world closely, however does not offer flexibility in terms of scalability and repeatability. Therefore, simulators are widely preferred for the performance analysis of these networks.

Simulators incorporate the component of mobility by using synthetic mobility models or real-world traces. Real-world traces are given preference in evaluations as they depict the realistic human mobility. However, the open source traces available for public use [2] are few 
and are scenario-specific. Though there are tools such as BonnMotion [3] to extract and modify these traces to a certain extent, they don't offer a wide variety of scenarios. Further, there is a lack of flexibility in customization and extension of these traces. On the other hand, synthetic mobility models are flexible and adaptable for setting up configurations based on the need.

Synthetic mobility models are developed based on human mobility studies and human movement in the real world. Some models have been built from traces, while few others are analytically modeled adhering to the observations of human behavior [4]. Thus, the variety of proposed synthetic mobility models ranges from random walks to map-based sophisticated models with pedestrian and vehicle movement. However, the implementation of mobility models in simulators for OppNets have been a few and requires significant attention as mobility is one of the major factors driving OppNet performance [5].

Of the widely used OppNet simulators, the ONE [6] has few implementations of highly used mobility models such as Random waypoint, Home Community Mobility Model (HCMM) and Working-Day Movement model (WDM) [7]. Adyton [8] supports only real-world traces which further need to be adapted as per the format required by Adyton. Opportunistic protocol Simulator (OPS) [9], currently the only simulator for OppNets based on OMNeT++ has linear movement, Random Walks (RW), Random Waypoint (RWP), Small Worlds In Motion (SWIM) [10] and provision to use traces [11]. However, RW and RWP are not realistic for modeling human mobility [12], as humans don't move in random directions with random speeds. The limited range of mobility models in OPS have restricted majority of the OppNet simulations to use traces or SWIM [13]. Further, the social groups based mobility and individual users' schedule based mobility are highly relevant in simulating OppNet forwarding protocols. As OM$\mathrm{NeT}++$ has the essential modules to implement new synthetic mobility models [14], [15], this paper brings forth the implementation of Community-based Mobility Model (CMM) [16] and probabilistic ORBIT [17], which would expand the horizon for upcoming OppNet simulations using OPS and OMNeT++.

The rest of the paper is organized as follows: Section 2 and Section 3 briefly gives an overview of CMM and ORBIT and their main functionalities. Section 4 presents the implementation of $\mathrm{CMM}$ and ORBIT in OMNeT++. Section 5 discusses the simulation results and validation of the implementations of those mobility models. Section 6 concludes the paper and addresses the future work.

\section{Community based Mobility Model}

The main idea of CMM is that the users with strong social network ties in real world tend to move in same geographical space [16] and hence, these social relationships can be used to model human mobility patterns. CMM models social relationships by using social network information to group users into communities. The social network information from the model is taken as input which is an interaction matrix ' $I$ ', and the entries of this matrix are weights ' $w$ ' of edges between two nodes $i$ and $j$. These weights are interaction indicators representing the strength of social interactions between the respective nodes. From the interaction matrix, a connectivity matrix is created by selecting those edge weights that cross a certain threshold $(>0.25)$. This value is used by authors in [16], based on their studies of the social network trace considered as input for the interaction matrix in their simulation.

Communities are detected by removing the nodes or links with high betweenness centrality. After removing a certain number of such links as given by a modularity factor $Q$ [16], the algorithm stops and by then, the nodes have been grouped into communities. The communities are assigned to a specific physical space in the simulation area. The whole simulation area is 
divided into equal size of non-overlapping grids and each community is assigned to an exclusive grid. The nodes belonging to the same community often move in same geographical space and the next target location of the nodes is based on the social attractivity of grids.

Social attractivity of a grid at any time is calculated by the sum of the interaction indicators of relationships between the node $i$ and all other nodes in that respective grid normalized by the total number of nodes in that square. The social attractivity of all the grids in the simulation area for a node $i$ is calculated and then the node $i$ moves to the grid with highest social attractivity. The next location of each node is selected in this manner which can be in the same grid or in a different grid depending on the social attractivity. The exact position in the selected grid is chosen randomly. There is a reconfiguration interval after which the communities are reset which captures the variations in human movement based on the time of the day.

\section{Orbit Mobility Model}

ORBIT mobility for MANETs is based on the sociological movement pattern of users [18]. Most of the users' movements are in a terrain consisting of certain locations or around these locations. ORBIT mobility model is not concerned about the exact coordinates or positions of the users at any instant of time. Instead, it focuses on identifying the approximate region where the user could be located at any given time. ORBIT assumes that users' spend most of their time only in few of the regions or locations in the simulation space and they do not visit every location in the simulation space. These particular locations are usually visited by users in some probabilistic manner which is determined by their individual schedules and hence, are a part of their mobility profiles. During weekdays and weekends, users usually might follow different movement patterns depending on their activities and individual schedules. This model is called ORBIT because it is based on the fact that users' typically move around these few locations in a sequence, however not necessarily the same sequence everyday.

ORBIT divides the simulation area into locations of different or equal sizes called hubs. A hub can be a classroom, a shopping mall, home, different rooms in a home, an entire street and so on. The concept of a hub can vary based on simulation scenario, however a simulation area is divided into hubs to capture the movement profile of the nodes. The total number of hubs in the simulation area is given by Total Hubs. A Hub list is associated with every user and the users orbit in these hubs in a partially deterministic sequence. The exact position of the users inside the hubs are not modeled by ORBIT, as its main goal is macro-level mobility. Every user or a node spends a certain amount of time in each hub given by Hub stay time. The hub list assigned to each node along with its hub stay times is named an Orbit. An orbit can be reset by an Orbit Timeout, after which the hub lists and hub stay times associated with nodes can be redefined.

Four different orbits are discussed in [18] such as random orbit, uniform orbit, restricted orbit and overlaid orbit. Additionally, probabilistic orbit [17] is discussed in the improvement of SOLAR. In this model, nodes move to different hubs with a probabilistic association. This can reflect in the selection of the next position and the hub stay time. Further, it allows the flexibility for the nodes to visit some hubs frequently and to stay in some hubs for a longer time.

Hub stay time restricts the overall time spent by a node in a hub. This parameter ensures that the nodes do not stay in the same hub for a significant portion of the simulation time, unless it is the requirement of the simulation scenario. Hub stay times can be different for different hubs and also for different nodes. Further, Orbit timeout ensures that nodes can follow different schedules during the same day or even week and this behaviour is incorporated in the mobility 


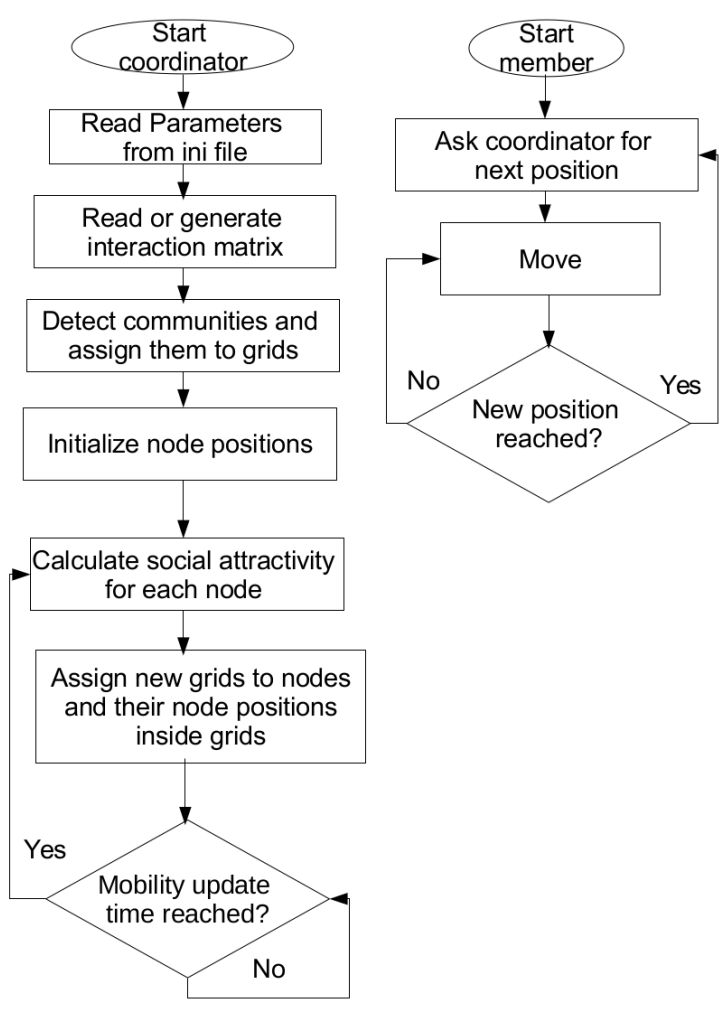

(a) CMM

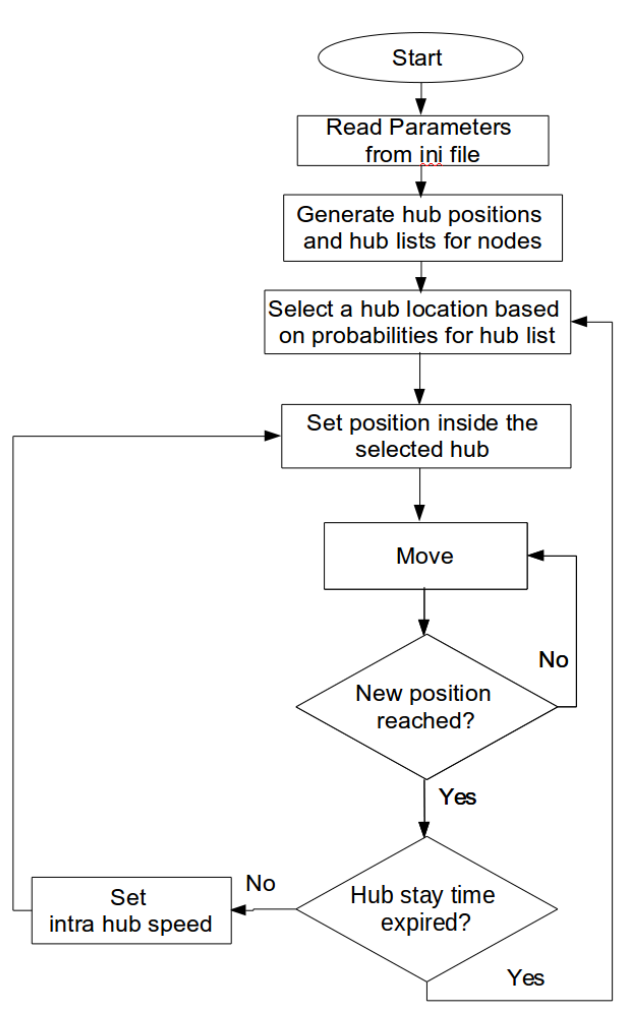

(b) ORBIT

Figure 1: Implementation flow chart of CMM and ORBIT

model in a generalized manner. For instance, all the nodes change their orbit movement after orbit timeout and this timeout is the same value for all the nodes in the simulation, which is not exactly the case in reality. The speed of a node within the same hub and between different hubs are given by Intra-hub speed and Inter-hub speed respectively, because nodes usually follow different modes of transport depending on the distance between their current and next target positions. However, this choice totally depends on the way hubs are partitioned.

\section{Implementation in $\mathrm{OMNeT}++$}

The CMM and ORBIT mobility model implemented in OMNeT++ uses the INET Mobility framework. The flow charts for both mobility model implementations are shown in Figure 1 and the implementations are available in Github ${ }^{1}$. With reference to the implementation, users, hosts and nodes are used interchangeably and refer to the same meaning. A position refers to the exact coordinates of a node and a location refers to an area where many nodes can be present. The implementations have two phases namely initialization phase and mobility phase.

\footnotetext{
${ }^{1}$ https://github.com/ComNets-Bremen/Mobility-Models
} 


\subsection{Community-based Mobility Model Implementation}

A separate folder named social is added in mobility of inet for CMM as CMM uses social network interactions and the interaction matrices are given as input files or generated randomly and placed in this folder.

CMM has two modules namely a CMM coordinator and CMM member. The CMM coordinator does the necessary preprocessing and calculating the grid locations for all nodes for every mobility update. The next target position of a node is influenced by the current locations of its social network contacts and hence, a node needs to know much more than its own current location to calculate the social attractivity of a grid. This operation is simplified by using a centralized module that keeps track of the locations of all the nodes in the simulation at any given time during the simulation. Thus, the CMM coordinator does all the pre-processing steps till mobility phase and particularly calculates the next target location and makes it available for the nodes. The CMM member module implements the mobility functions of a node. A CMM member asks the CMM coordinator for the next target location and uses the function move() to reach the next location. The next target location can be in the current residing grid or in a different grid based on the social attractivity of grids as described in section 2 .

\subsubsection{Initialization Phase}

All the tasks in initialization phase is done byspeeds CMM coordinator.

Loading or creating the interaction matrix: It is a matrix depicting the probabilities of interaction between every pair of nodes. It is a $n \times n$ matrix where $n$ is the number of nodes. It can be generated with random probabilities or the matrix can be read from a file. If the interaction matrix is to be generated randomly, then the function setInteractionMatrix() performs this operation. If the interaction matrix is to be read from a file, then the function loadInteractionMatrix() performs this operation. The option of reading the interaction matrix from a file enables the users to load predefined interaction matrices from real-world traces.

Forming communities: From the interaction matrix, the CMM coordinator makes all the values above a certain threshold $(>0.6)$ as 1 leading to the creation of the connectivity matrix. The authors in [16] used a real-world trace which was not available to us and hence, we generated a random interaction matrix. In order to avoid having a complete random community assignment, we selected a higher threshold of 0.6 as we required communities with greater interaction compared to the interaction levels selected by authors in [16]. The entries of connectivity matrix gives the nodes that interact with each other more often.

Following the connectivity matrix, communities are formed by removing the node with highest number of connections and all the nodes connected to the node with highest connectivity. The group of these severed nodes become a community. This procedure is repeated and communities are formed until all the nodes are covered. If there is a lone node, it becomes a community on its own. The function createCMMCommunities() makes these communities and the output is the number of communities and the community to which a node belongs.

Assigning communities to grids: Each community is assigned to a random space in the simulation area. The simulation area is divided into number of grids. The grid size is read from the ini file by the parameter gridLength. The simulation area is divided into equal number 
of non-overlapping grids which are squares of length and breadth given by grid length. The function assignCommunitiesToGrids() assigns the communities to grids randomly.

\subsubsection{Mobility Phase}

In the mobility phase, the next target position of each node is calculated based on the social attractivity factor. The function calculateSocialAttractivity() in CMM coordinator calculates the social attractivity of grids for each node and returns the community with highest social attractivity for each node. The output of this function tells the node which grid it should move to based on the locations of its social network connections. The node then uses setTargetPosition() and selects a random position in the target grid, sets its speed and then uses the move() function to move linearly to the exact position in the target grid. The CMM member performs the actual mobility in the mobility phase, though the location decision is performed in the CMM coordinator.

\subsection{ORBIT Implementation in OMNeT++}

ORBIT mobility reads the following parameters from ini file and sets up the hub locations and hub positions in the initialization phase. The parameters are taken from [18], where this model was proposed.

\subsubsection{Initialization Phase}

Number of Hubs: The total number of hubs in the given simulation area is set by this parameter numberOfHubs. It was set to 10 in our simulation.

Hub stay time: The time spent by a node in a hub is given by hubStayTime. This time can vary for different nodes in different hubs. It was set as a value between 50 and 100 seconds.

Intra-hub speed: Nodes use intraSpeed to move if the next position is within the same hub. It was set as a value between 1 - 3 meters/second considering pedestrians.

Inter-hub speed: Nodes use interSpeed when moving to a different hub than the current hub location. It was set as a value between $1-6$ meters/second considering pedestrians and bicycle users.

Hub list: Every node has a hubList which can be generated by a matrix or read from a file. A node moves between the hubs assigned to it. It does not move to any hub that is outside of its hublist.

Hub size: The area of each hub is setup inside the simulation region as given by hubSize. Hub size in our simulation was $200 \mathrm{~m} \times 200 \mathrm{~m}$.

Orbit timeout: The hub lists and hub sizes are reset after this timeout period orbitTimeout. In our simulation, it was set to 8 hours. 


\subsubsection{Mobility Phase}

The node decides its next location based on its hub list and the probabilities associated with the hubs in the hub list. The node generates a random number between 0 and 1 and uses it as a threshold to selects the hub with probability close to the obtained threshold. The node sets a random position inside the selected hub and moves linearly to that position using move(). The node selects intraHubSpeed or interHubSpeed based on the choice of the next hub. Till the expiration of hubStayTime, it moves to a random position in the same hub for every mobility update. After the hubStayTime expires, the node moves to a different hub in the consequent mobility update.

\section{$5 \quad$ Simulation and Results}

The ORBIT and CMM implementations in OMNeT++ were simulated with the setup as given in Table 1. CMM and ORBIT simulations were reset after a reconfiguration interval or timeout period of 8 hours. Hence, the behaviour of the nodes were refreshed thrice in total simulation time. The objective here is to capture the different movement patterns of the nodes within a single day.

\begin{tabular}{|c|c|c|c|}
\hline Parameter & RWP & CMM & ORBIT \\
\hline Nodes & 100 & 100 & 100 \\
\hline Area & $5 \mathrm{~km} \times 5 \mathrm{~km}$ & $5 \mathrm{~km} \times 5 \mathrm{~km}$ & $5 \mathrm{~km} \times 5 \mathrm{~km}$ \\
\hline Simulation time & 24 hours & 24 hours & 24 hours \\
\hline Community size / Hub size & - & $200 \mathrm{~m} \times 200 \mathrm{~m}$ & $200 \mathrm{~m} \times 200 \mathrm{~m}$ \\
\hline Speed & $1-6$ meters $/ \mathrm{sec}$ & $1-6$ meters $/ \mathrm{sec}$ & $\begin{array}{c}\text { intra-hub speed: } 1-3 \mathrm{~meters} / \mathrm{sec} \\
\text { inter-hub speed: } 1-6 \text { meters } / \mathrm{sec}\end{array}$ \\
\hline Mobility update interval & 1 second & 1 second & 1 second \\
\hline Hub stay time & - & - & 50 - 100 seconds \\
\hline Timeout / Reconfiguration interval & - & 8 hours & 8 hours \\
\hline
\end{tabular}

Table 1: Simulation Configuration for RWP, CMM and ORBIT mobility models

\begin{tabular}{|c|c|c|c|c|}
\hline Mobility model & Number of events & Simulation time & Real-time & Completion \\
\hline CMM & 8690795 & 86400 seconds & 41.7975 seconds & $100 \%$ \\
\hline ORBIT & 8675916 & 86400 seconds & 30.7734 seconds & $100 \%$ \\
\hline RWP & 8644602 & 86400 seconds & 30.2608 seconds & $100 \%$ \\
\hline
\end{tabular}

Table 2: Simulation Performance of CMM, ORBIT and RWP 


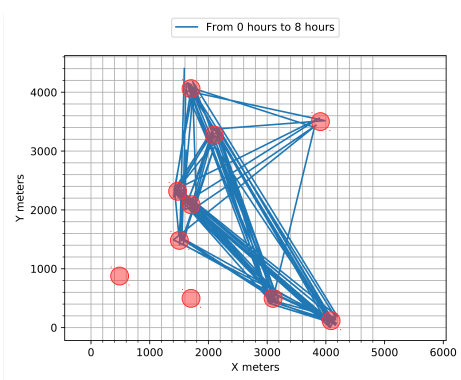

(a) ORBIT

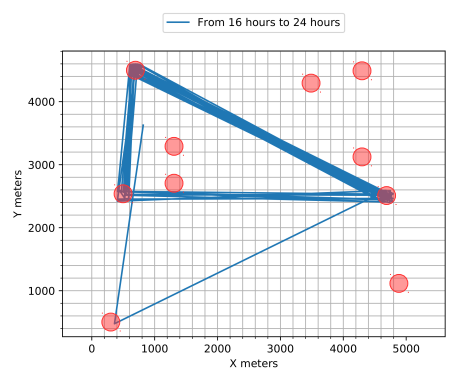

(c) ORBIT

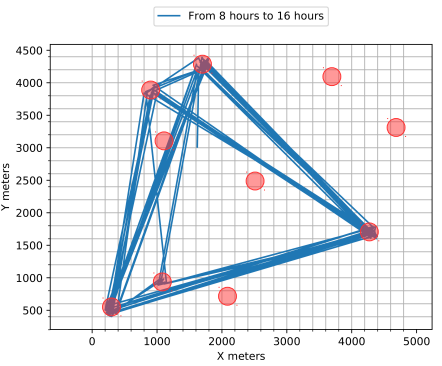

(b) ORBIT

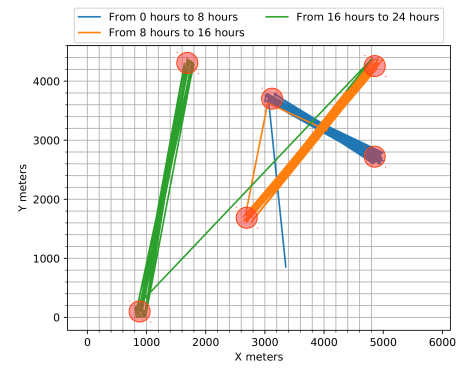

(d) CMM

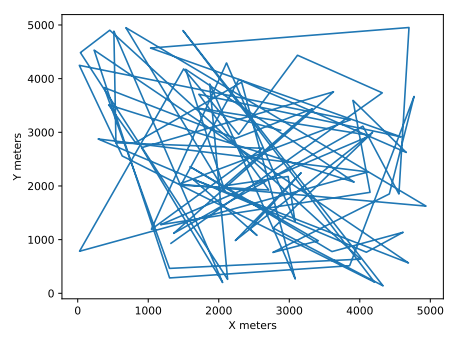

(e) Random-waypoint

Figure 2: Trajectories of ORBIT, CMM and RWP mobility models

These simulations test the basic operation of CMM and ORBIT models, and the results are compared with RWP in terms of mobility trajectory of a single node as shown in Figure 2. The shaded circles in Figure 2(d) shows the different communities for CMM and the shaded circles in Figure 2(c) shows the different hubs for ORBIT respectively. The trajectories in Figure 2(d) verifies that the nodes in CMM move along with their social network contacts frequently, spending more time in some of the grids and not visiting some grids at all. In ORBIT, the nodes visit certain hubs frequently and does not visit other hubs as seen in Figure 2(c). On the other hand, the nodes in RWP move over the entire simulation area.

The comparison of simulation performance for the three mobility models is shown in Table 2 . CMM has highest number of events due to the calculation of social attractivity for all the nodes for every mobility update. ORBIT, on the other hand has to perform selection of hubs for every mobility update and hence, it has second highest number of events next to CMM. However, RWP 


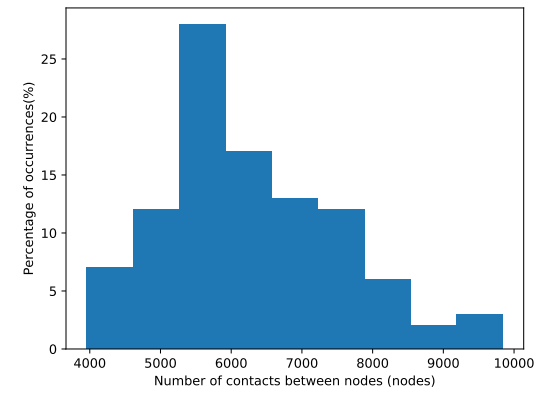

(a) Total number of contacts between nodes

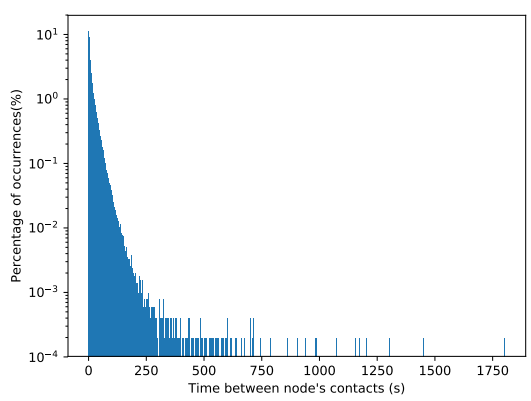

(c) Time interval between contact times

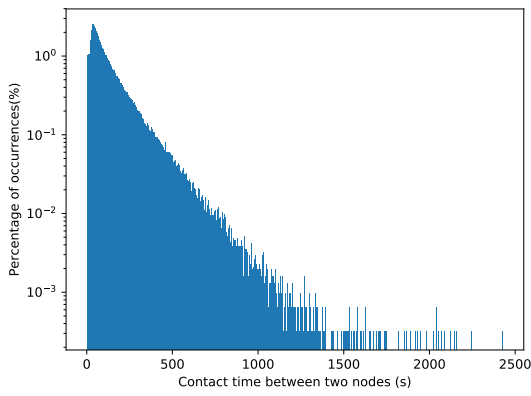

(b) Contact times between nodes

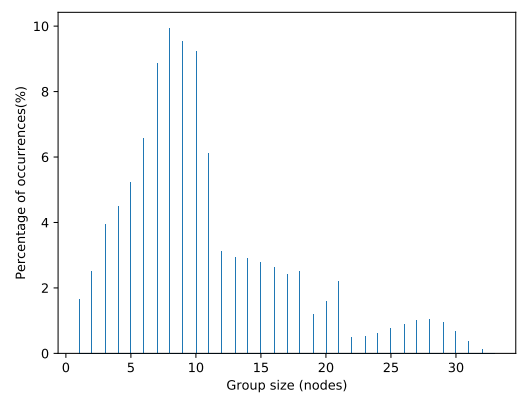

(d) Community size

Figure 3: Community-based Mobility Model (CMM)

randomly selects a random position in the simulation area for every node during the mobility update and thus, has the fewer events of all the three mobility models under consideration in this paper.

Further, the contact times and number of contacts for CMM, ORBIT and RWP with the chosen simulation configuration is shown in Figure 3, Figure 4 and Figure 5. Figure 3(a) shows that 25 percentage of the nodes in CMM had approximately 6000 contacts during the entire simulation time as the nodes are highly likely to move together with nodes of same community rather than meeting new contacts. The nodes in CMM has an average of 6290 contacts throughout the simulation. Figure 3(b) shows that the contact times between nodes or plainly contact times in CMM is distributed between 1 and 1500 seconds. The high contact times in CMM are due to the fact that nodes with strong social ties move within same geographical space and hence, stay often in contact. Figure 3(c) shows that within 1 to 250 seconds, the nodes meet a neighbor and hence, this represents the time between contact times. Time between contact times refers to the time interval during which the nodes were not able to meet any contacts. Figure 3(d) shows that the community size in CMM varies from 1 to 30 during the simulation. For 10 percentage of the simulation, the number of nodes in a community was 7 .

Figure 4(a) shows that 25 percentage of the nodes in ORBIT has 11000 contacts as the nodes move between different hubs as compared to CMM. Though this movement pattern depends on the hub list assigned to each node, the number of contacts in ORBIT is generally higher than 




(a) Total number of contacts between nodes

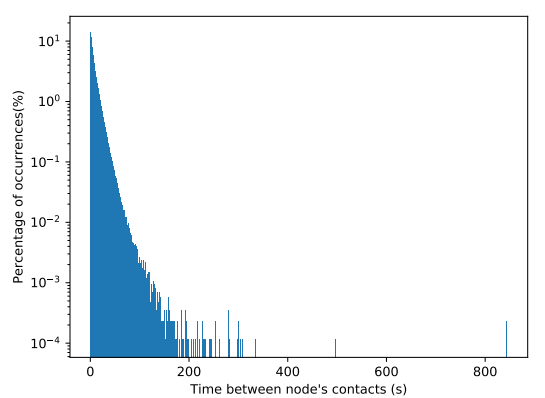

(c) Time interval between contact times

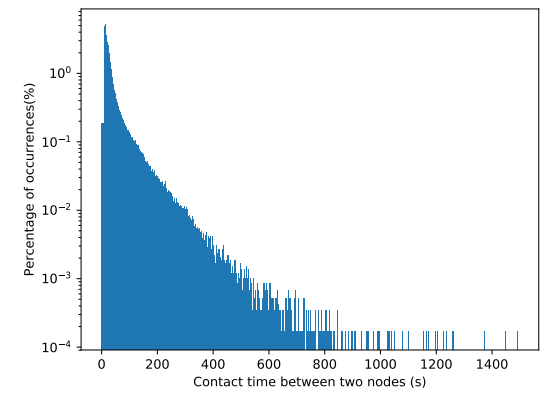

(b) Contact times between nodes

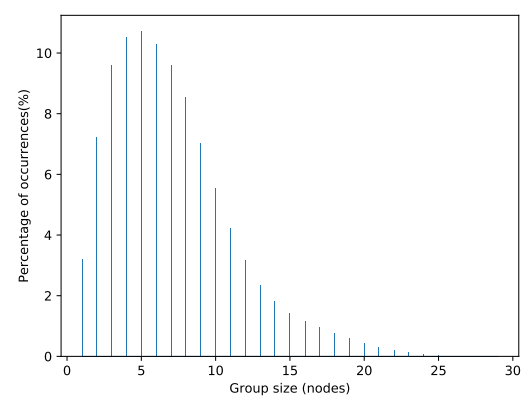

(d) Hub size

Figure 4: ORBIT Mobility

CMM as the node movements in ORBIT are not dependent on other nodes. Hence, the nodes using ORBIT is more likely to make more contacts compared to CMM. However, the contact times from Figure 4(b) are in the range of 1 to 450 seconds which is lesser than CMM, as the node movements in ORBIT are not dependent on other nodes. Hence, the nodes in ORBIT are not likely to be in contact often for a longer contact duration in comparison with CMM. Figure 4(c) shows the time interval between contact times is 1 to 200 seconds and Figure 4(d) shows the hub size. The hub size was approximately 6 for 10 percentage of the simulation time. Figure 5 shows the results of RWP to compare the with the results of CMM and ORBIT in Figure 3 and Figure 4. From Figure 5, it can be seen that the number of contacts in RWP is lesser compared to CMM and ORBIT as the movement is more random and hence, does not support meeting contacts on purpose. The contact times and time between contacts are also dependent on this random movement behaviour of RWP.

\section{Conclusion}

The work in this paper presented the implementations of CMM and ORBIT mobility models in OMNeT++. The implementations use the mobility architecture of OMNeT++ and the operations follow the original models proposed in [16] and [17], [18]. The performance of these models have been verified and their mobility patterns show that the nodes in CMM move 


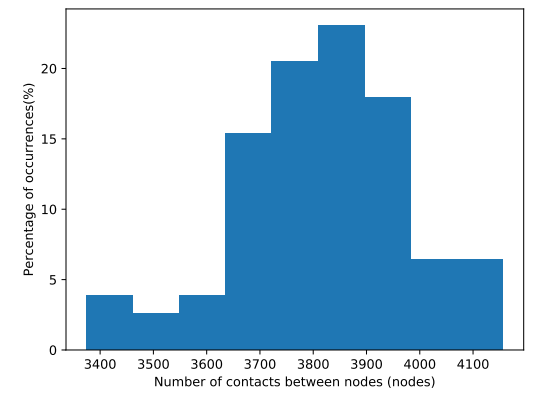

(a) Total number of contacts between nodes

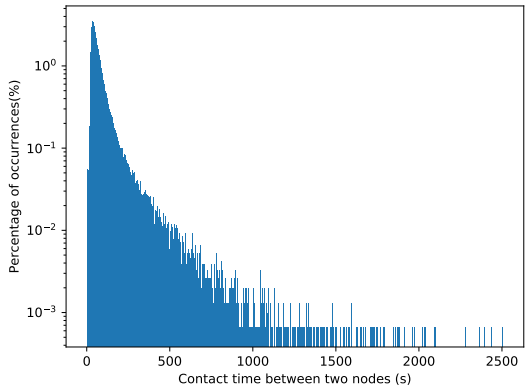

(b) Contact times between nodes

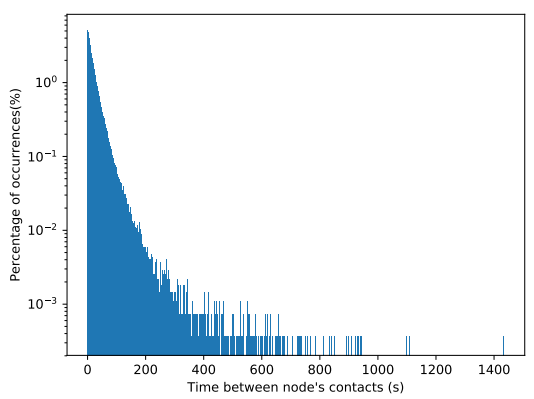

(c) Time interval between contact times

Figure 5: Random-Waypoint Mobility (RWP)

between very few communities and the nodes in ORBIT move only between the hubs in their hub list. The contact durations and the number of contacts are also influenced by these behaviours, which also aligns with the proposed pattern in [16] and [17]. The current implementations are evaluated only using the simulation parameters and thus, there is a potential to evaluate these implementations using traces in the future work.

\section{References}

[1] Vinícius FS Mota, Felipe D Cunha, Daniel F Macedo, José MS Nogueira, and Antonio AF Loureiro. Protocols, mobility models and tools in opportunistic networks: A survey. Computer Communications, 48:5-19, 2014.

[2] Tristan Henderson and David Kotz. \{CRAWDAD\} wireless network data citation bibliography. 2015.

[3] Nils Aschenbruck, Raphael Ernst, Elmar Gerhards-Padilla, and Matthias Schwamborn. Bonnmotion: a mobility scenario generation and analysis tool. In Proceedings of the 3rd international ICST conference on simulation tools and techniques, page 51. ICST (Institute for Computer Sciences, Social-Informatics and Telecommunications Engineering), 2010.

[4] Dmytro Karamshuk, Chiara Boldrini, Marco Conti, and Andrea Passarella. Human mobility models for opportunistic networks. IEEE Communications Magazine, 49(12):157-165, 2011. 
[5] Suvadip Batabyal and Parama Bhaumik. Mobility models, traces and impact of mobility on opportunistic routing algorithms: A survey. IEEE Communications Surveys \&5 Tutorials, 17(3):16791707, 2015.

[6] Ari Keränen, Jörg Ott, and Teemu Kärkkäinen. The one simulator for dtn protocol evaluation. In Proceedings of the 2nd international conference on simulation tools and techniques, page 55. ICST (Institute for Computer Sciences, Social-Informatics and Telecommunications Engineering), 2009.

[7] Frans Ekman, Ari Keränen, Jouni Karvo, and Jörg Ott. Working day movement model. In Proceedings of the 1st ACM SIGMOBILE workshop on Mobility models, pages 33-40. ACM, 2008.

[8] N Papanikos, DG Akestoridis, and E Papapetrou. Adyton: A network simulator for opportunistic networks, 2015.

[9] Asanga Udugama, Anna Förster, Jens Dede, Vishnupriya Kuppusamy, and Anas Bin Muslim. Opportunistic networking protocol simulator for omnet++. arXiv preprint arXiv:1709.02210, 2017.

[10] Asanga Udugama, Behruz Khalilov, Anas Bin Muslim, and Anna Förster. Implementation of the swim mobility model in omnet++. arXiv preprint arXiv:1609.05199, 2016.

[11] Liu Sang, Vishnupriya Kuppusamy, Anna Förster, Asanga Udugama, and Ju Liu. Validating contact times extracted from mobility traces. In International Conference on Ad-Hoc Networks and Wireless, pages 239-252. Springer, 2017.

[12] Jens Dede, Anna Förster, Enrique Hernández-Orallo, Jorge Herrera-Tapia, Koojana Kuladinithi, Vishnupriya Kuppusamy, Pietro Manzoni, Anas bin Muslim, Asanga Udugama, and Zeynep Vatandas. Simulating opportunistic networks: Survey and future directions. IEEE Communications Surveys $\&$ Tutorials, 20(2):1547-1573, 2018.

[13] Vishnupriya Kuppusamy. Performance analysis of epidemic routing in destination-less oppnets. In 2018 IEEE 19th International Symposium on" A World of Wireless, Mobile and Multimedia Networks"(WoWMoM), pages 01-03. IEEE, 2018.

[14] Behruz Khalilov, Anna Förster, and Asanga Udugama. Radio irregularity model in omnet++. arXiv preprint arXiv:1709.07035, 2017.

[15] Anna Förster, Anas Bin Muslim, and Asanga Udugama. Reactive user behavior and mobility models. arXiv preprint arXiv:1709.06395, 2017.

[16] Mirco Musolesi and Cecilia Mascolo. A community based mobility model for ad hoc network research. In Proceedings of the 2nd international workshop on Multi-hop ad hoc networks: from theory to reality, pages 31-38. ACM, 2006.

[17] Joy Ghosh, Hung Q Ngo, and Chunming Qiao. Mobility profile based routing within intermittently connected mobile ad hoc networks (icman). In Proceedings of the 2006 international conference on Wireless communications and mobile computing, pages 551-556. ACM, 2006.

[18] Joy Ghosh, Sumesh J Philip, and Chunming Qiao. Sociological orbit aware location approximation and routing (solar) in manet. Ad Hoc Networks, 5(2):189-209, 2007. 